\title{
An Agent Cellular Residential Mobility Model : From Functional and Conceptual View
}

\author{
Elarbi Elalaouy*, Khadija Rhoulami, Moulay Driss Rahmani \\ LRIT associated unit to CNRST (URAC ${ }^{\circ} 29$ ), Faculty of Science, Mohammed V University in Rabat \\ 4 Av.Ibn Battouta B.P. 1014 RP, 10006 Rabat, Morocco
}

\begin{abstract}
Residential mobility is of great challenge to sustainable cities. Developing computer models based simulation could be a powerful tool to support informing urban decisions especially with the fact that half of the world's population now lives in cities. The present paper presents our detailed model of residential mobility which use an alliance Multi-agent systems and Cellular automata (MAS-CA) approach. Conventional Urban modelling approaches will be presented firstly with a distinct light sheded on the alliance CA-MAS approach. The model will be then exposed in its two functional and conceptual views. At the end, results of a scenario of growth population is simulated and discussed. Results of the simulation shows significant conformity with the underlying model hypothesis.
\end{abstract}

Keywords-Residential mobility; land use change; computer model; multi-agent systems; cellular automata

\section{INTRODUCTION}

Residential mobility is an urban phenomenon that has been the subject of research in several disciplines, starting with sociology, demography, geography, planning and urban planning [1] [2] [3] [4]. It is characterized by residential movements, both individual movements (decision to move and housing choice) and aggregated ones (population flows) of an urban population. Residential mobility is mainly concerned with intra-urban mobility taking place within an urban agglomeration, without taking into account the mobility over longer distances (example of interurban mobility).

Studies of residential mobility have among others the following objectives : 1) understanding individual and aggregate residential choices of city dwellers; 2) studying spatial structures resulting from the residential choices of the population; 3) anticipating and predicting residential dynamics; 4) testing the possible evolutions in the face of modifications of the infrastructure such as insertion of building zones, industrial zones, etc; 5) meeting needs of the population in terms of housing and transport infrastructure.

Conventional studies of residential mobility carried out by state institutions (which are statistical in nature) are important [5]. They make it possible to quantify the phenomenon of residential mobility, to clarify its causes and present effects. However, the decisions and urban actions derived from these studies can only be gradual or progressive as they are a reaction to a present urban reality. The current state of the question shows that conventional studies cannot alone achieve objectives (3-4-5) since these objectives require predictive and simulation capabilities that are deficient to conventional studies.
Issues of environmental and sustainable development of cities, advances in computer science have allowed the creation of a new movement of modeling and simulation of urban dynamics. This new urban dynamics modeling and simulation movement focuses on the development of computer simulation models that harness data on population and urban infrastructure, predict mutual dynamics of population and infrastructure, and analyze results of the simulation in order to understanding urban systems and support with decision making.

In this article, we will present firstly four main urban modelling approaches that have been used in the literature; we shed then the light on the alliance CA-MAS approach. Secondly, we will outline our developed residential mobility model from a functional and conceptual view. Finally, results are then presented to highlighting usefulness of this CA-MAS modeling approach for supporting decisions making and urban planning.

\section{URBAN MODELLING APPROACHES}

Modeling and simulation of urban dynamics, including residential mobility, has been marked by the use of several approaches. We present four main modeling approaches.

\section{A. The Regional Approach}

The regional approach is an approach originated from economics. This approach considers the city as divided into several administrative areas. Each zone includes data on infrastructure (land-use distributions, housing categories, housing prices, etc.) and on population (socio-economic and cultural group distributions, etc.), mainly coming from statistics on these administrative areas [1]. These areas are the basic units of the regional models that attempt to manage the flows between zones. The objects exchanged can be population, commodity, capital, etc. Zones have weights that depend on their scales.

\section{B. The Statistical Approach}

The statistical approach is based on the analysis and exploitation of general population censuses and household surveys [5].. This approach attempts to measure urban phenomena (such as residential mobility, urban growth, urbanization, etc.), describe the evolution of these phenomena at a spatio-temporal scales, and prepare cross-tabulations for example of behaviors of household mobility and housing characteristics (location, housing type, occupancy status) with those of individuals and households (age, occupation, income level, etc.) 


\section{Cellular Automata Approach}

The cellular automata approach is a bottom-up approach. It divides the urban space into a grid of cells. Each cell has a state among a finite set of system states. future states depend on transition rules based on a local spatio-temporal neighborhood. The system is homogeneous in the sense that the set of states is the same for each cell and the same transition rules are applied to each cell. Time advances in discrete steps and the application of transition rules on all cells can be synchronous or asynchronous [6].

Conventional CA models use neighborhood-based rules with local scope. New applications of CA models are becoming more and more free of this specificity. They use rules whose neighborhood is defined by a distance to the relevant cell [7]. For example, in a city, when looking for a new home, the exploration of a household can be done in the radius of its current home. This radius can cover gradually a district, a town or the entire city.

CA models use either IF-THEN transition rules or probabilistic transition rules. The IF-THEN approach consists of using simple rules implemented as conditions to model the dynamics of the system [8]. This approach, according to our knowledge, is the least used in contemporary time in urban modeling. The probabilistic approach, on the other hand, is becoming increasingly important in modeling spatial dynamics. The Markov chain models incorporated within CA models are an example of models that introduce probabilistic transition rules [9]. The authors of [10] reported that an approach combining $\mathrm{CA}$ and Markov chains benefiting from the potentials of both methods is a preferred approach. The cellular automaton represents the spatial distribution of the land use of an urban space, and above it, the Markov chains predict the total rate of land use change from the past changes.

Cellular modeling techniques are the core of many spatial dynamics models. The use of these techniques for geographic process modeling dates back to the nineties. Advanced CA models currently exist for modeling and simulating spatial dynamics, evacuation systems, river basin dynamics, forest fire spreading [11] [12] [13] [14]. In particular, these cellular techniques have also contributed to the modeling and simulation of residential mobility. Among the first theoretical works that simulated the residential movements of individuals in an urban space are the works of James M. Sadoka and the works of Thomas Schelling as reported in [6]. The two authors designed a cellular space inhabited by a population belonging to two social groups and then tried following their own models of transition rules to simulate manually the residential displacements of individuals over cellular space.

Both models are very simple in construction but it inspired researchers in modeling and simulation of residential mobility [1] [2] [15]. These latter models inherit Sadoka and Schelling's idea of conception, which allows on one side to place each individual entity, which can be either an individual or a household, in a cell that is used as housing, and on the other hand, to use the transition rules as a proxy to trigger the decision-making of individual entities. For example, in the work [2] a model of residential mobility simulation based on cellular automaton was designed. The population is organized into individual households each of which occupies a cell of the automaton which represents the inhabited dwelling. The transition rules of the CA model make it possible to govern households in their housing change decisions. Emerging residential dynamics are generated from the interaction of changes in the social structure of households and the positive or negative influence of the neighborhood. This example sheds light on how the transition rules can be used as proxy between the cellular automaton that represents the studied city and the population that lives there.

Modeling efforts, mainly using the cellular automaton approach, for the modeling of the resulting spatial dynamics in addition to the decisions of the urban actors, succeed as the work [2] demonstrates. However, this cellular method succeeds only if the unit of analysis is in harmony with the used cellular arrangement, the decisions of the urban actors are at the same level of organization and the local neighborhood affects the different actors with a simple and uniform way. When the actors are not related to the locations in an intrinsic manner of the cells of the cellular automaton, the neighborhood relations of the conventional cellular automaton cannot reflect the neighborhood relations of the urban actors. The solution to this problem depends on techniques that use non-uniform transition rules and that can dynamically change the configurations of cell connections. Because these techniques far exceed the capabilities of conventional CA models, conventional CA methods cannot be widely adapted to modeling urban dynamics. A model of urban dynamics may require multiple urban actors arranged in space, agents arranged at different scales of organizations (e.g., population, urban managers), or agents controlling certain parts of urban space.

In summary, CA techniques have clearly demonstrated their ability to model the spatial aspects of urban dynamics, yet they face challenges when considering the decisions of urban actors, especially when these actors are working at different organizations scales. To overcome these challenges, it is necessary to implement complex and multiscale transition rule sets that apply to different groups of cells. Even if this solution is possible, the deviations from generic CA will be to the detriment of giving up the potential benefits of generic CA. In particular, In order to ensure reuse, and extensibility in other applications and domains through modeling and simulation platforms, it is necessary that the form of the CA preserves as many features of generic models as possible.

\section{Multi-Agent Systems Approach}

While the cellular automata approach focuses on the representation of space and its transition, the multi-agent approach is interested in the representation of actors so-called agents, their actions and interactions in the system. Agents are the fundamental elements in multi-agent models. Several characteristics define the agents : they are autonomous; they share an environment through communication and interaction; and they make decisions that bind behavior to the environment. Agents have been used to represent various entities such as atoms in physics, cells in biology, animals, robots, humans and organizations. Autonomy means that agents act and interact with their total will, even when these agents are dominated from an external entity, these agents do not perform actions unless they have chosen them. Wooldridge defines intelligent 
agents as being able to act flexibly [16]. This imply that agents are objective-oriented in their actions and that they are able to interact with other agents and a common environment, which is everything outside the agents. In an urban modeling context, a shared environment can be an urban space where the actions of each agent can affect those of others. It can also be a land market through which agents buy and sell housing and land.

Agents must perform actions that result from a thought process that links their goals to the environment through their behavior. This thinking process, also known as the cognition model, can range from simple stimulus-response decisions to the point where agents become proactive and make decisions. An autonomous agent needs, at a minimum, to perceive his environment and react accordingly to the perceived knowledge. The reaction can be considered as a cognitive model, in the sense that it is the response of agents to change. Some models of cognition go beyond reaction, they consider agents as possessors of rationality and perfect analytical capacity that allow them to solve the complex problems of mathematical optimization. These models trying to make the agents perfectly rational are contradicted by the experimental proofs. It is an open question if these models of perfect rationality are appropriate to multi-agent urban models, knowing the interdependencies and interrelations in its systems. The difficult recognition of the complex environment in which human decision-making occurs has led to shift to a new variant of agent models with reduced rationality. Instead of implementing a solution that completely predicts the future states of the system they are part of, agents use heuristic classification and prediction methods. Bayesian networks, neural networks and genetic algorithms are examples of methods that have been used to design agent cognition models in some urban systems [17] [18] [19].

The applications of the agent approach for designing complex systems are not to be demonstrated, however, residential mobility applications designed solely based on the agent approach are not up to the required height since the agent approach is not originally interested in modeling spatial environments. On the other hand, there is some modeling work in residential modeling that has opted for the use of the agent approach. These residential mobility agent models, do not integrate urban space as environment, they do not have spatial representation as reported by [20]. To represent the agent environment, these agent models incorporate land market models. The work of [21] illustrates this kind of model through a model of multi-agent simulation of displacements of the population in a mono-centric urban city. The model does not incorporate the urban space in which the population moves, it uses a reduced grid of two dimensions, which makes it possible by differentiating housing units by their distance to the center of the city, as well as by amenities by location.

These models rarely encountered in literature, described a research track that begins to be forgotten, in contemporary times, for two main reasons. The first reason is that these models do not include spatial information of the city such as equipment units, hospitals, schools etc. The second reason, even if they are agent based models, they do not deal with individual residential behaviors including mobility decision, and housing choice decision. This research, which uses the agent approach in residential mobility modeling, shows, on the one hand, that the agent approach is useful for the design of urban actors, in particular households, but on the other hand, they illuminate well that this agent approach does not have the means to represent the urban space, place where interactions of human agents happen.

\section{PROPOSED RESIDENTIAL MOBILITY MODEL}

\section{A. The Alliance Approach of CA\&MAS}

The alliance approach of CA-MAS is a recent approach for modeling urban dynamics that are characterized by both societal and spatial dynamics. Despite its novelty, it has in its repertoire various applications from urban modeling as it is an emerging approach of two approaches each having a great maturity in the areas of modeling and simulation of complex systems. Torrens reports that this new approach is useful for the development of planning support tools, with the ability to simulate individual households and built-up areas in a realistic, dynamic and flexible way [22]. It are the limitations of the previously presented approaches that are causing the movement towards this new modeling and simulation approach. The lack of dynamic representation in urban simulations, the lack of detail in spatio-socio-economic representations and the lack of inclusion of bottom-up processes in complex systems are the major disadvantages of conventional regional models [22].

Regional models operate at a very high level. In fact, when heterogeneity and interaction at a local level are important in urban dynamics, regional models will have a very limited capacity to explain these dynamics. Statistical models provide a sophisticated view of multi-dimensional decision indicators. However, these models condense the information into estimated parameters that represent the average effects of the available data. Indeed, these models can be useful for the projection of dynamics and spatial interactions only for phenomena guaranteeing stationarity and uniformity in space and time. Although cellular models offer greater flexibility for the representation of spatio-temporal dynamics, they have limited means to represent urban actors. Cellular models do not fail to model urban actors only when they are arranged in the same way of cells of the cellular automaton. None of the previous approaches can effectively represent the different decisions of urban actors that are characterized by autonomy, heterogeneity and decentralization except multi-agent approach. But the latter does not have the means to represent the urban space where interaction of human agents happen.

The alliance MAS-CA approach addresses limitations of each of the previous approaches. In particular, this alliance approach has a great capacity for modeling the urban dynamics resulting from the interactions of human and spatial entities. The approach benefits from the complementary advantages of both MAS and CA approaches. The MAS approach can represent urban actors, their behaviors and their interactions with each other and with their environment. The CA approach can represent spatial units like houses, apartments, schools, etc.. The MAS-CA approach benefits also from the theoretical underpinnings of both approaches. In the SMA Framework, spatial dynamics, the development of built-up areas and the creation of vacant housing are considered emerging dynamics 
of the actions of urban actors, particularly the population living in the urban area; In the CA framework, spatial dynamics are considered as resulting dynamics of statistical concepts of transition rules (eg., Markov chain concepts) applied to a series of cellular maps of the studied region. These two theoretical foundations make it possible to see the city in these two societal and spatial aspects and can bring more representation to the intrinsic processes that govern urban areas.

The new alliance approach MAS\&CA creates a new motivation for the modeling and simulation of residential mobility. Although urban models, particularly those of residential mobility, are not simple in design and development, researchers are increasingly relying on previous approaches and are increasingly involved in this new framework of the alliance of MAS-CA approaches. Among the most important models, we could find [1] [20] [4]. These residential mobility models are generally based on similar theoretical frameworks (with of course, specific features of each model) derived mainly from advances in the social and environmental sciences. These theoretical frameworks represent residential dynamics according to a bottom-up approach called the "stressresistance" approach, which sees residential dynamics as the result of the residential actions of different households in the population. In its standard version (see Figure 1), households take two basic steps, the first stage concerns the decision to leave the dwelling, the second stage concerns the decision to reside in a new location [1]. In the first stage, households estimate the "stress" of moving by comparing the current stress to the desired residential situation; if the stress is high enough, they decide to change housing. In the second stage, those who are willing to move estimate the "resistance" to relocate by comparing available alternatives to their current home, and then decide to either move to one of the alternatives or stay in their current home. He continues to say that this approach has several names "dissatisfaction", "utility" and "residential dissonance". The urban system, in these models of residential mobility, includes urban space and population particularly individual households which perform residential actions namely the mobility decision and the housing choice decision.

These residential mobility models, based on the stressresistance approach, are more or less characterized by the following modules: 1) a module for representing urban infrastructure; 2) a spatial dynamics module; 3) a household representation module 4) a households mobility module 5) a housing choice module 6) and a population projection module.

\section{B. Context and Hypothesis}

The residential mobility simulation model that we propose is part of this new trend of combining CA-MAS approaches for the modeling and simulation of urban systems. This model represents the urban space by a two-dimensional cellular automaton. Thus, urban space consist of a set of juxtaposed uniform spatial units. The model represents the households constituting the population of the urban space by mobile agents having the mobility capacity in the urban space. Household agents use socio-economic data from household surveys. Agents make decisions independently.

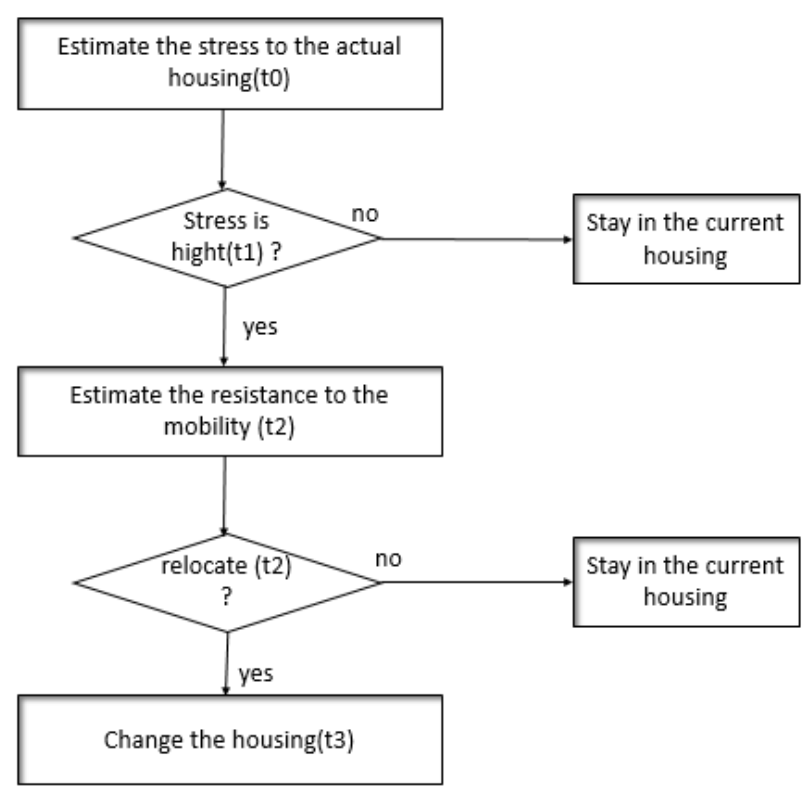

Fig. 1. The stress-resistance approach, $t_{1}<t_{2}<t_{3}$ indicates consecutive moments of the temporal sequence of a household related to its residential actions (adapted from [1])

Each agent decides for himself whether he wants to change housing or not. If this decision is positive, he starts looking for a new home that suits him. These agents are cognitive and thus have a reasoning ability about their mobility and housing choices. In fact, each agent has a mobility decision function designed using a probabilistic model based on mathematical concepts of Bayesian networks. It also has a function of choosing the best housing from a list of suitable housing to his desire. Thus, an agent explores the urban space starting with the nearest neighborhood. In case of need, the householder increases his field of research until finding suitable housing.

The model also governs the dynamics of the urban space through a complementary layer of Markov chain and Cellular automaton. The transition rules of the cellular automaton are then governed according to a probabilistic model using mathematical concepts of Markov chain. The model also governs the growth of the population through a population projection sub-model. On an annual basis, a new generation of households is generated from the previous generation using demographic indicators such as birth rate, member departure rate, marriage rate, divorce rate and expectancy of life.

\section{The Contribution of the Model}

The objective of our model is the simulation of residential mobility and the associated urban growth over a horizon of tenyears. The city being the place where the two phenomena run, is the result of residential choices at the individual level of households. The urban system as a whole consist of an urban space, a set of households, a set of dwellings and a time dimension. Urban space including housing is approached by a grid of cellular automaton. The households making up the population are approached by a multi-agent system.

The model of residential mobility we are presenting is an extension of two-research work [23] [24]. The first research work focus on the functional aspect of the proposed residential 
mobility model, and describe its mathematical equations. The second research work focus on the conceptual aspects of the proposed residential mobility model, which consists of UML 2.0 based models. The objectif of the UML 2.0 modeling is to boost the development phase of residential mobility Framework. This research work bring these two aspects together in order to show the proposed residential mobility model as entirely as possible. In addition, the present work describe, from an algorithmic view, the four functional models, which represent the core of the proposed residential mobility model.

\section{DETAILED DESCRIPTION OF THE MODEL}

\section{A. The Functional View}

The RM model consists mainly of four mathematical models as below. This new description of the functional view has origin in the research work [23] which describe in detail the mathematical models. Figure 2 depicts the architecture of the RM model and its components.

- The mobility decision model: which uses a classification technique to find out whether each given household with given characteristics has a propensity for changing housing or not. The propensity for mobility noted $P^{t}($ relocation $\mid K)$, where $\mathrm{t}$ is the time, $\mathrm{K}$ the vector of household characteristics, is calculated as well detailed in [23]. Figure 5 shows the corresponding algorithm.

- The Housing Choice Model: allows each household to explore the neighborhood gradually (rank 1, rank 2, etc.), look for potential dwellings, and ultimately choose the most suitable dwelling to the household. The equation denoted $P_{C_{i j}}$ is used to calculate the attraction potential of a cell $C_{i j}$. Figure 7 shows the corresponding algorithm.

- Spatial prediction model: which makes it possible to predict spatial dynamics, this one uses either spatial prediction techniques or manual configurations that can be parameterized in the RM Framework. In our model, the Markovian equation that governs the spatial transition is denoted $P\left(C_{i j}^{t+1}=\mathrm{e}\right)$, it designates the probability that the spatial unit $C_{i j}$ transit to the state e at time $\mathrm{t}+1$. The Figure 4 shows the corresponding algorithm

- The population projection model: which allows by means of demographic rates such as the rate of birth, divorce, marriage, departure etc, to project the next population from a given population. This model could use projection technique or manual configurations introduced in the Framework. The Figure 6 shows the corresponding algorithm.

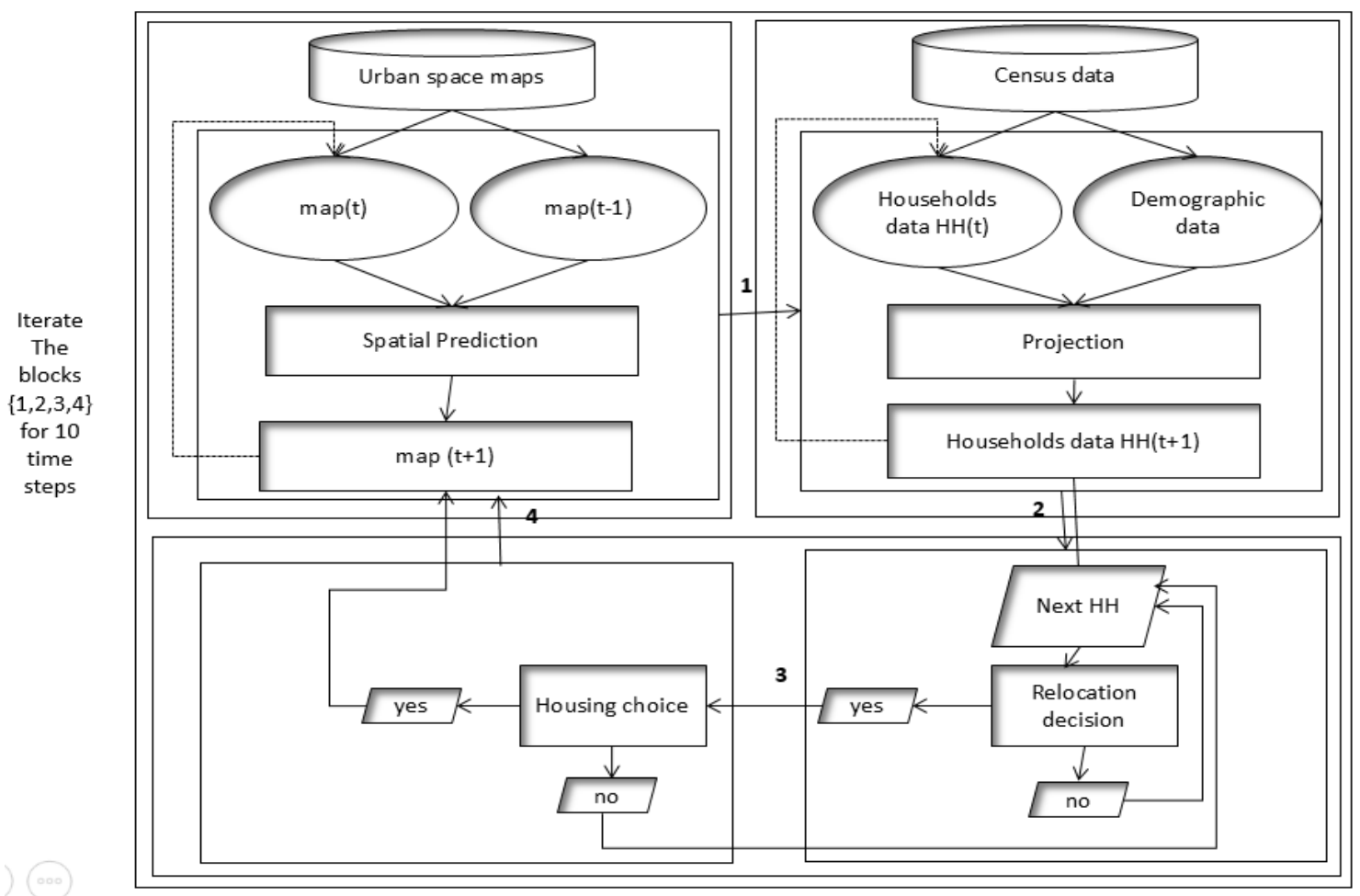

Fig. 2. Architecture of the residential mobility model gathering the above described sub-models.

\section{B. The Conceptual View}

The model consists mainly of four conceptual models designed using the UML 2.0 modeling language. This new description of the conceptual view has its origin in the modeling work [24] which describe in detail these conceptual models. The Figure 3 expose a light weighted combination of the four models in one model.

- The model of the urban space: represents the urban space by means of a cellular automaton approach. The 
urban space is therfore considered as a set of juxtaposed spatial cells having states representing the soil occupancy.

- The population model: represents the population of households and their characteristics, in the simulation framework; the population are approached by a multiagent system.

- The model of population dynamics: allows archiving of population generations over a calendar of years. This model allow on one hand to trace the demographic evolution of the population and on the other hand observe the movements of households in the urban space.

- The model of urban space dynamics: which traces the evolution of urban space during a calendar of years of simulation. This traceability will thus make it possible to observe the evolution of built-up areas in the studied urban space.

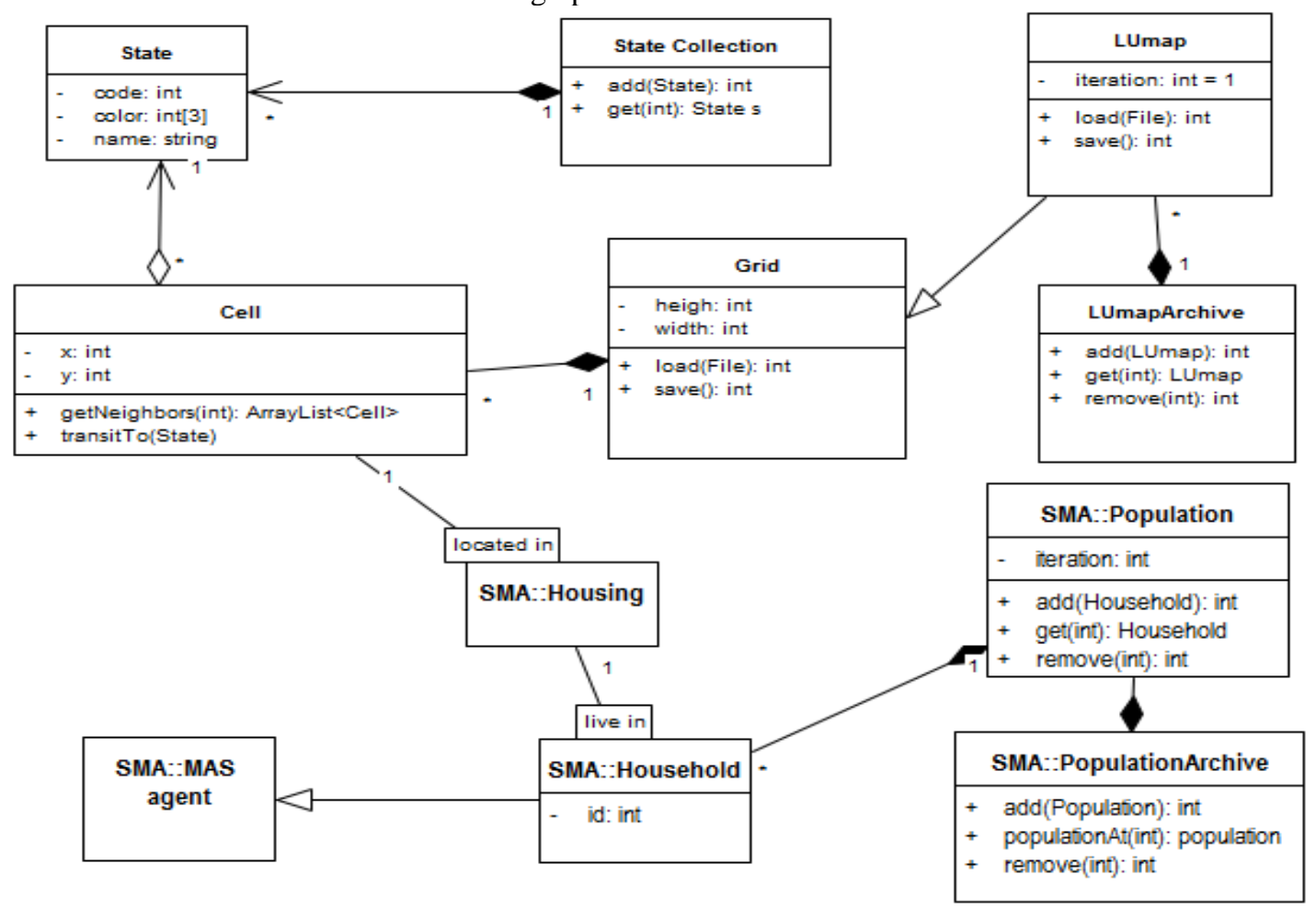

Fig. 3. The dynamic model CA-MAS of residential mobility

\section{Data of the Model}

The model uses three types of input data, with which begin the simulation of residential mobility, namely maps of urban space, household survey data, and initialization parameters:

- Household data: represent socio-economic data of all households in the urban area. Households are classified according to their household type (Family With Children, Family Without Children, Single Family and Single Person). Households have also characteristics such as occupancy status, age of householdholder and average income. Households are also classified as owners and renters according to their occupancy status.

- Maps of urban space: represent the land use of urban space. The model requires two reference maps at time $t_{0}$ and $t_{1}$. It is from these two maps that the map at time $t_{2}$ is predicted, and so on. The preparation the urban space map require different spatial data layers of the studied area in particular (road and rail infrastructure, urban planning, building areas etc).
Maps of urban space are designed according to a predefined format and use static and dynamic states of land use : road or rail network, river or lakes, green space or forest, commercial building and equipment building. The elementary dynamic states are owned single house, rented apartment, rented single house, rented apartment, and building area

- Initialization parameters: represent demographic rates and other simulation parameters. Demographic rates, such as birth rate, departure rate, divorce rate, marriage rate and life expectancy govern the life cycle of households. In other words, the transition, for example, from single-person households to a Family Without Child households is governed by the marriage rate, and so on. There are other parameters which control the simulation such as the total duration of the simulation, the spatial resolution of the cellular automaton and the environmental quality of the housing type. 


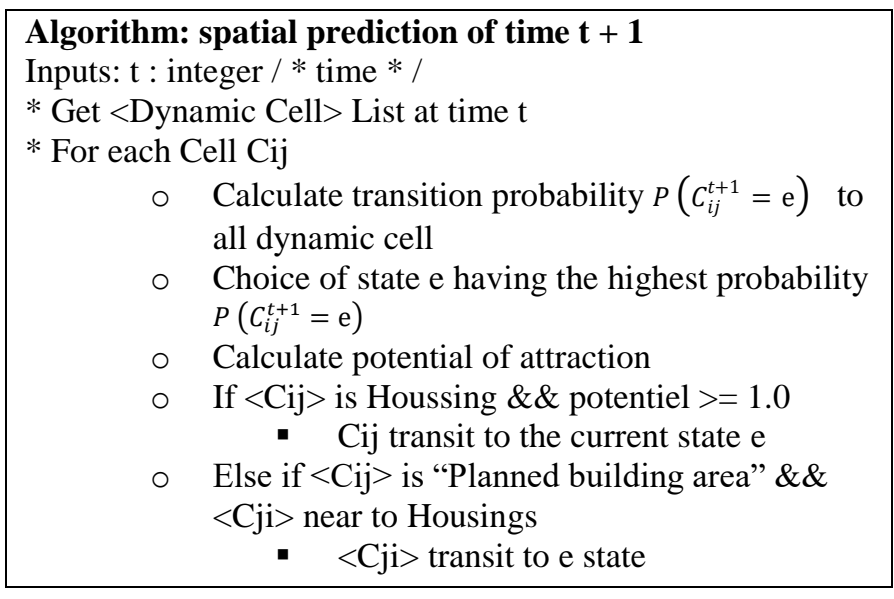

Fig. 4. Spatial prediction algorithm

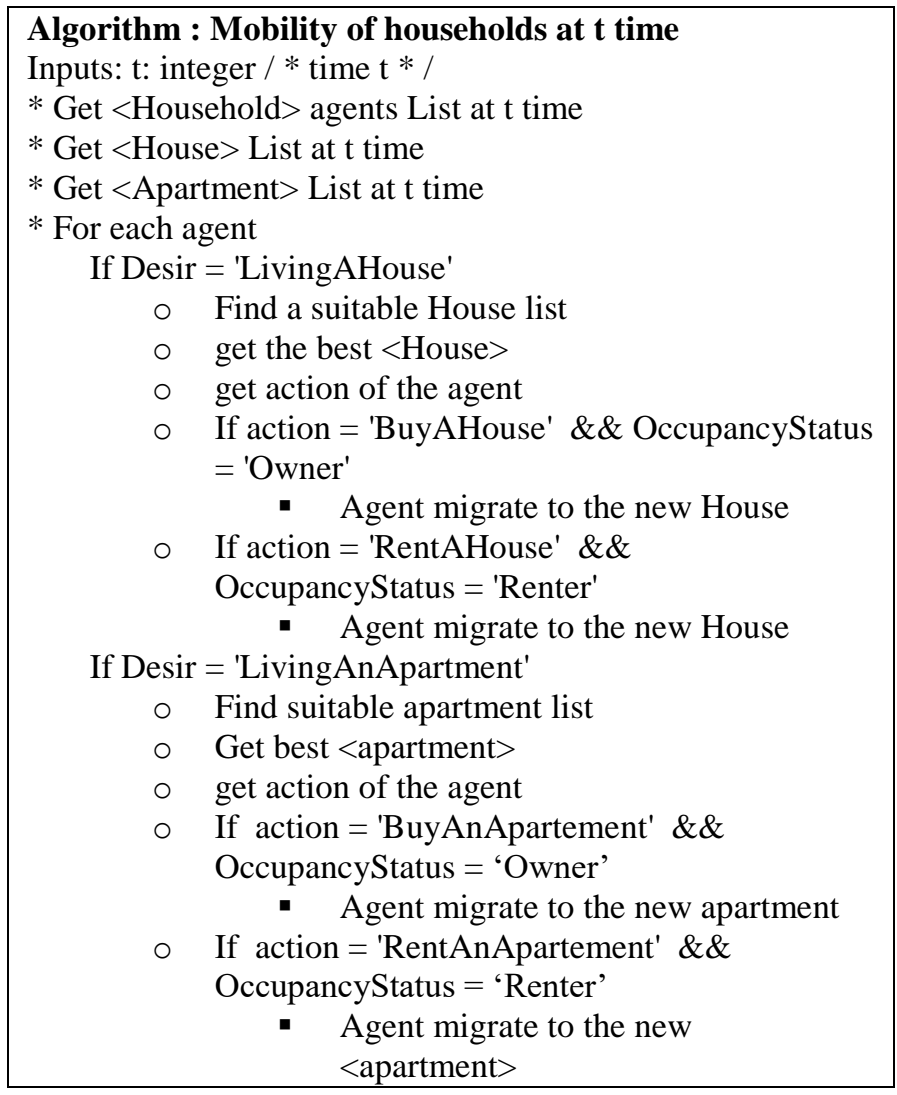

Fig. 5. Algorithm of Mobility of household agent a t time

$$
\begin{aligned}
& \text { Effectif }_{\mathrm{PS}}^{\mathrm{t}+1}+=\text { Effectif }_{\mathrm{PS}}^{\mathrm{t}} *\left(1-\mathbf{T}_{\text {mariage }}\right) \\
& \text { Effectif }_{\mathrm{PS}}^{\mathrm{t}+1}+=\left(\text { Effectif }_{\mathrm{FAE}}^{\mathrm{t}}+\text { Effectif }_{\mathrm{FM}}^{\mathrm{t}}\right) * \mathbf{T}_{\text {départ }} \\
& \text { Effectif }_{\mathrm{FSE}}^{\mathrm{t}+1}+=\frac{1}{2} * \text { Effectif }_{\mathrm{PS}}^{\mathrm{t}} * \mathbf{T}_{\text {mariage }} \\
& \text { Effectif }_{\mathrm{FSE}}^{\mathrm{t}+1}+=\text { Effectif }_{\mathrm{FSE}}^{\mathrm{t}} *\left(1-\mathbf{T}_{\text {divorce }}\right) \\
& \text { Effectif }_{\mathrm{PS}}^{\mathrm{t}+1}+=2 * \text { Effectif }_{\mathrm{FSE}}^{\mathrm{t}} * \mathbf{T}_{\text {divorce }}
\end{aligned}
$$

$$
\begin{aligned}
& \text { Effectif }_{\mathrm{FAE}}^{\mathrm{t}+1}+=\text { Effectif }_{\mathrm{FAE}}^{\mathrm{t}} *\left(1-\mathbf{T}_{\text {divorce }}\right) \\
& \text { Effectif }_{\mathrm{PS}}^{\mathrm{t}+1}+=\text { Effectif }_{\mathrm{FAE}}^{\mathrm{t}} * \mathbf{T}_{\text {divorce }} \\
& \text { Effectif }_{\mathrm{FM}}^{\mathrm{t}+1}+=\text { Effectif }_{\mathrm{FAE}}^{\mathrm{t}} * \mathbf{T}_{\text {divorce }} \\
& \text { Effectif }_{\mathrm{FAE}}^{\mathrm{t}+1}+=\text { Effectif }_{\mathrm{FSE}}^{\mathrm{t}} * \mathbf{T}_{\text {naissance }}
\end{aligned}
$$

Fig. 6. Algorithmic equations of the population projection model

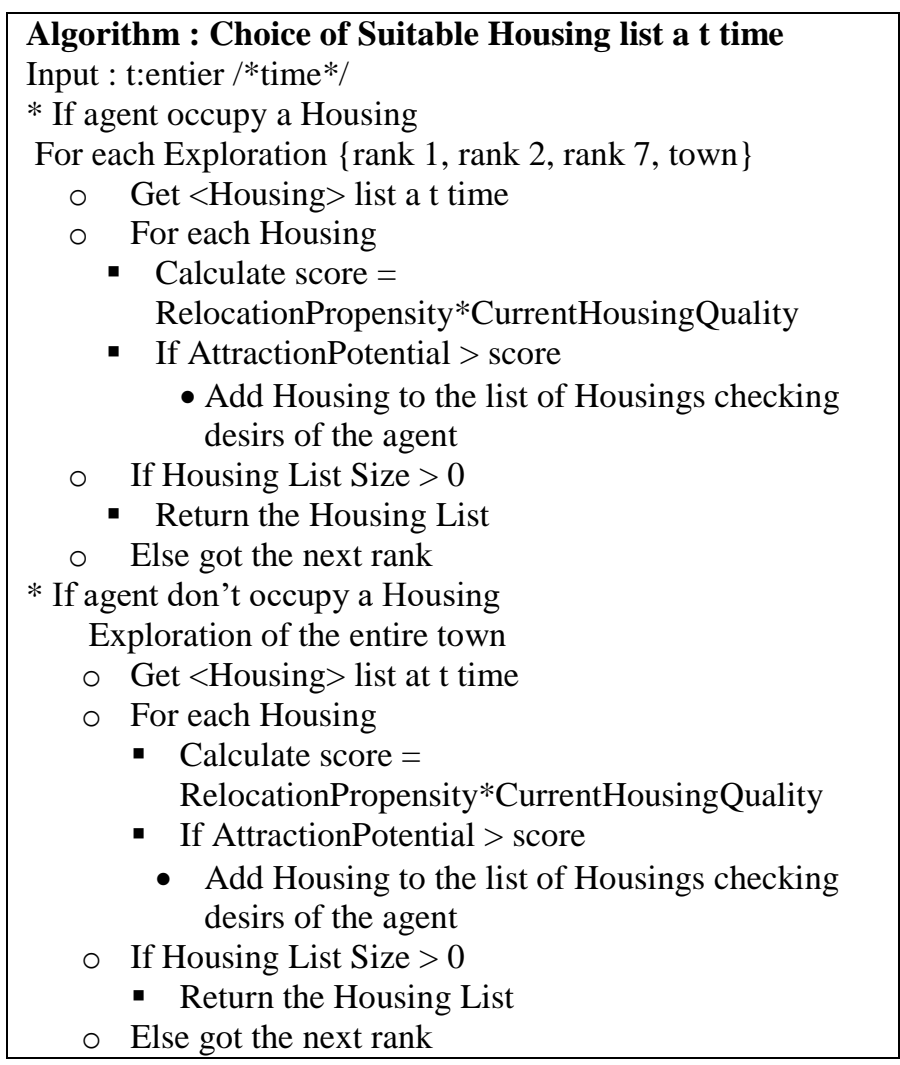

Fig. 7. Algorithm of choice of suitable housing at $t$ time.

\section{RESULTS AND DISCUSSION}

The simulation starts by parametring the number of households, housings and maps that represents initial data of the studied area. Real data of a Salé City could not be acquired entirely, so we will expose here some results of our model of residential mobility we have developed by simulating a growth population scenario that uses a small synthetic City. The number of housings and households is respectively 216 and 160; birth rate, departure rate, divorce rate, marriage rate and life expectancy have respectively $0.7,0.3,0.5,0.6$, and 70 . A number of housings are vacant at the simulation beginning.

Households have features which form their distinct profiles and which subsequently affect their residential choices notably residential mobility and housing choice. Population evolves yearly in terms of household's number, using the demographic projection model based on statistical data. Calibration of household data had performed with a distribution of housing types by household categories similarly to [20]. 
From the maps at Figure 8, the initial transition matrix and the probability distribution are computed. We then compute transition matrix and probability distribution at next stage based on Markov chain principles, and use it subsequently to predict yearly land use change in terms of built up areas as done by the spatial prediction model.
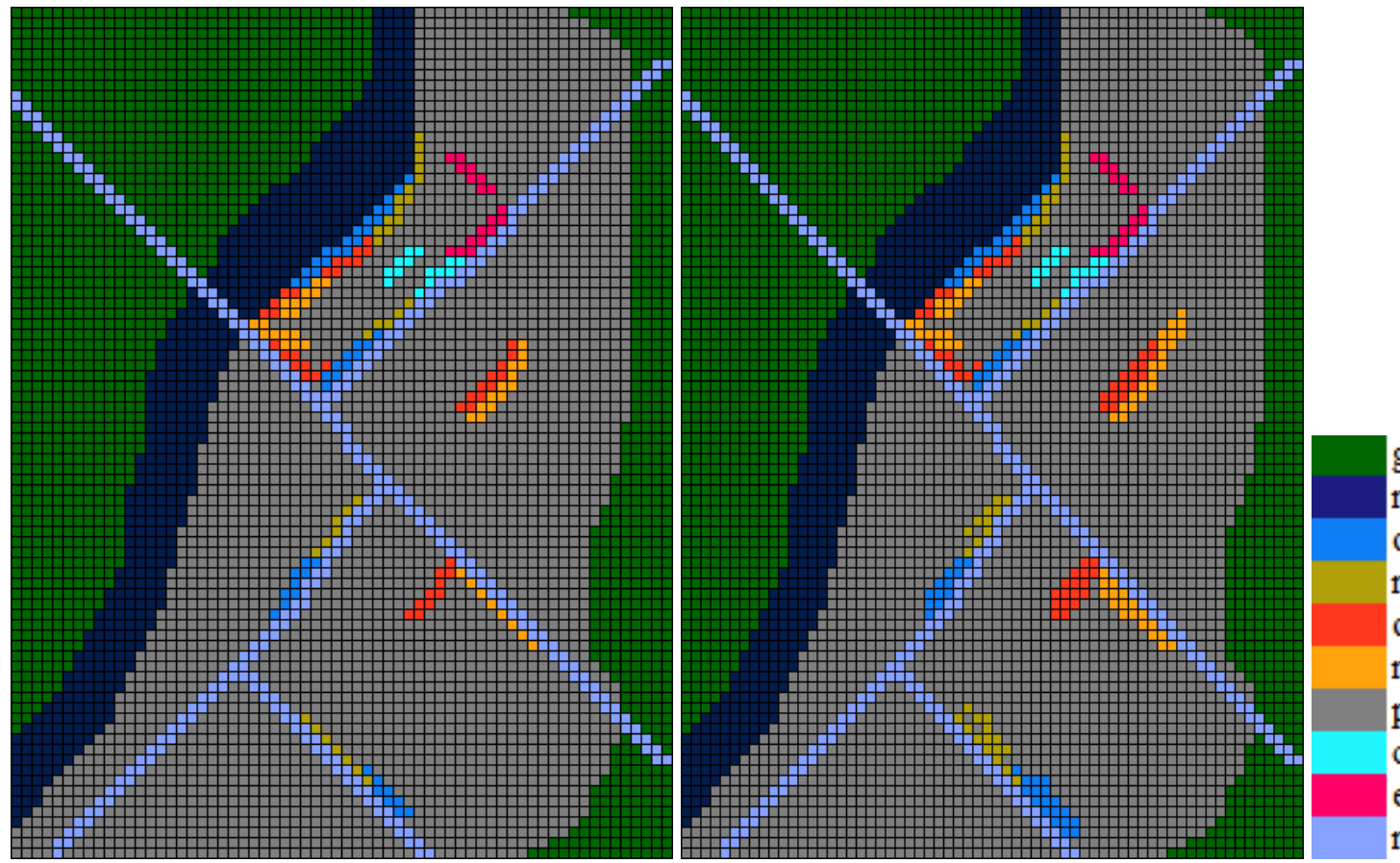

green space or forest river or lakes owned apartment rented apartment owned house rented house planned building area commercial building equipments building

Fig. 8. City maps 2006 and 2007 (from left to right)

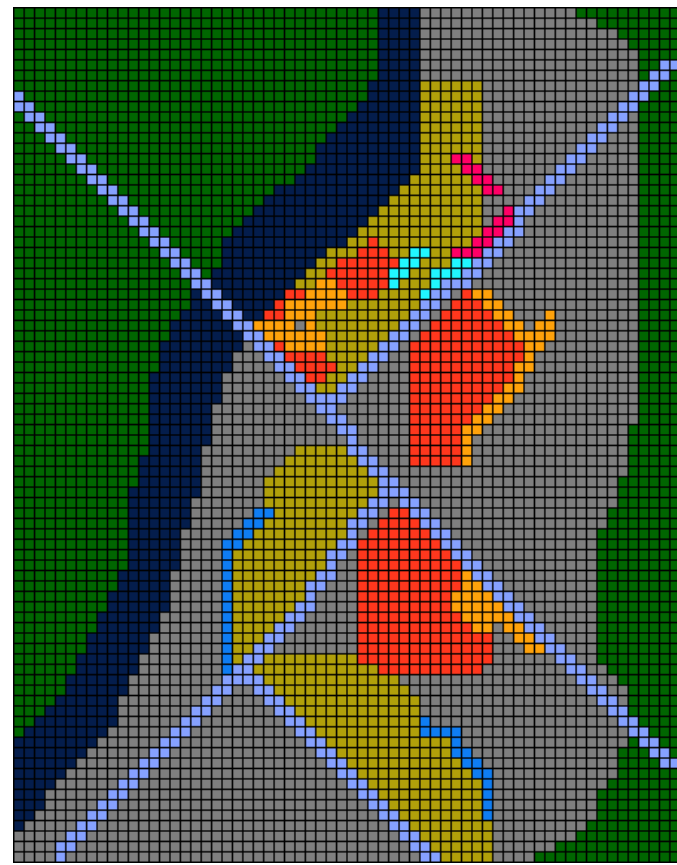

(a)

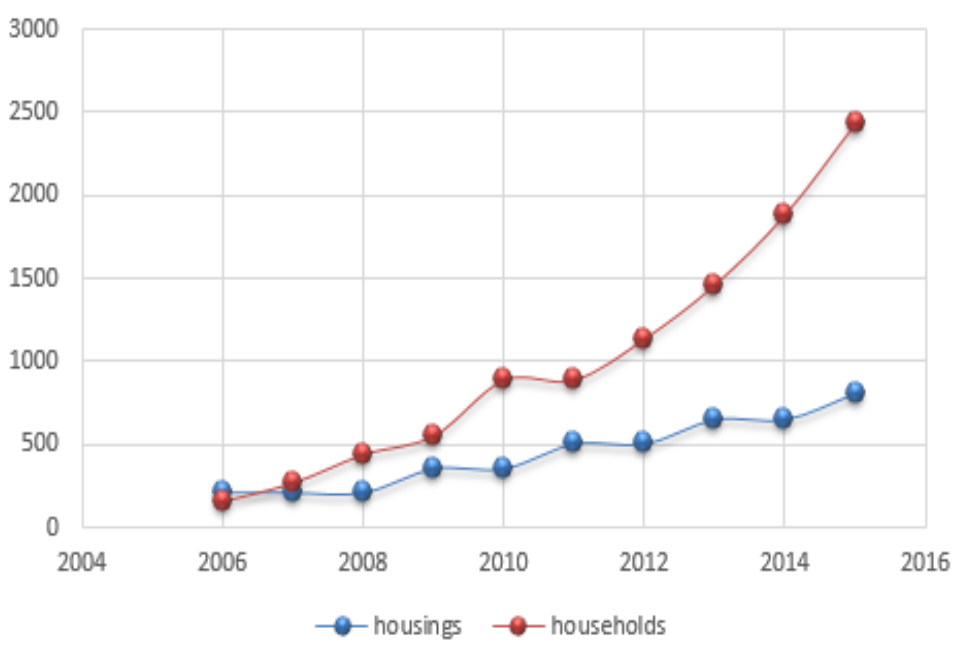

(b)

Fig. 9. (a) Expected 2015 built-up areas, (b) housings and hiuseholds evolution

After development of built-up areas, households (including those generated by the population projection model) begin their residential choices. New generated Households search for vacant housings using the housing choice model that allows them to explore gradually housings in their neighborhoods. Other households verify their residential satisfaction, if they are not satisfied with their actual housing; they use the housing choice model to look for new residence. Thus, some households move to their new housing, while others do not because they could not find housings that correspond to their intention. 
The model iterate yearly over a calendar of ten years. But lower duration could be also specified. Figure 9 expose the predicted built-up areas and the evolution of households and housings numbers. At the initial year of the simulation, we note that number of households is less than housings. Since the second year, in contrast to housings, household's number grow quickly. This result show a gap between population growth and constructed houses, which means that the annual built-up development rate is too slow regarding population growth rate. Such result, besides others ones that could be extracted by analyzing simulation outputs, could support decisions makers, to prepare effective urban decisions ( e.g., around housing needs, urban regeneration, households' energy consumption etc).

\section{CONCLUSION AND PERSPECTIVES}

The proposed model could be used to give insights into the dynamics of residential mobility and the linked land use dynamic. We used an interesting alliance CA-MAS approach for designing and implementing the proposed model. In the Framework of the model, the City is considered as an emergent and auto-organized system where the global consequences are results of a set of individual behavior of the smallest units notably households and housings. The proposed model, as being presented, is exposed in functional and conceptual aspects in order to show it as entirely as possible. We presented also, the core of the proposed residential mobility model from an algorithmic view, which could bring also sufficient information on the implementation phase.

The evaluation of the model have taken place as it has developed. Two levels of evaluation were conducted: human evaluation focused on observing predicted land use maps; and an operational evaluation that focused on observing the input outputs of the model's internal variables as well as the progress of the simulation between the different time steps. The twolevel evaluation demonstrated a significant degree of consistency between model assumptions and simulation results.

A third level of evaluation, of operational type, could be envisaged when acquiring all real data of an effective City. This type of evaluation could use confusion matrix and kappa index and play a support for Framework comparaison.

\section{REFERENCES}

[1] I. Benenson, «Agent-based modeling: From individual residential choice to urban residential dynamics,» Spatially integrated social science: Examples in best practice, vol. 42, pp. 67-95, 2004.

[2] V. Dabbaghian, P. Jackson, V. Spicer et K. Wuschke, «A cellular automata model on residential migration in response to neighborhood social dynamics,» Mathematical and Computer Modelling, vol. 52, pp. 1752-1762, 2010.

[3] V. Gaube et A. Remesch, «Impact of urban planning on household's residential decisions: An agent-based simulation model for Vienna,» Environmental modelling \\& software, vol. 45, pp. 92-103, 2013.

[4] V. Gaube, A. Remesch et B. Smetschka, «Planning, Residential Decisions and Energy Use in Vienna,» chez Social Ecology, Springer, 2016, pp. 489-503.

[5] S. Fol, Y. Miot et C. Vignal, MOBILITÉS RÉSIDENTIELLES, TERRITOIRES ET POLITIQUES PUBLIQUES, Presses universitaires du Septentrion, 2014
[6] R. Hegselmann et A. Flache, «Understanding complex social dynamics: A plea for cellular automata based modelling,» Journal of Artificial Societies and Social Simulation, vol. 1, p. 1, 1998.

[7] K. R. E. Elalaouy et M. D. Rahmani, «Cellular Automata: From Theoretical Concepts to Urban Modeling a New Methodology and Perspective,» Journal of Engineering and Applied Sciences,, vol. 13, pp. 4260-4266, 2018.

[8] E. Dubos-Paillard, Y. Guermond et P. Langlois, «Analyse de l'évolution urbaine par automate cellulaire. Le modèle SpaCelle,» L g\{l'e\} ographique, vol. 32, pp. 357-378, 2003.

[9] H. S. Moghadam et M. Helbich, «Spatiotemporal urbanization processes in the megacity of Mumbai, India: A Markov chains-cellular automata urban growth model,» Applied Geography, vol. 40, pp. 140-149, 2013.

[10] Y. Du, W. Wen, F. Cao et M. Ji, «A case-based reasoning approach for land use change prediction,» Expert Systems with Applications, vol. 37, pp. 5745-5750, 2010.

[11] J. P. Antoni, V. Judge, G. Vuidel et O. Klein, «Constraint Cellular Automata for Urban Development Simulation: An Application to the Strasbourg-Kehl Cross-Border Area,» chez Geomatic Approaches for Modeling Land Change Scenarios, M. T. Camacho Olmedo, M. Paegelow, J. Mas et F. Escobar, Éds., Cham, : Springer International Publishing, 2018, pp. 293-306.

[12] J. Ji, L. Lu, Z. Jin, S. Wei et L. Ni, «A cellular automata model for highdensity crowd evacuation using triangle grids,» Physica A: Statistical Mechanics and its Applications, 2018.

[13] B. K. Pandey et D. Khare, «Analyzing and modeling of a large river basin dynamics applying integrated cellular automata and Markov model,» Environmental Earth Sciences, vol. 76, p. 779, 2017.

[14] Z. Zheng, W. Huang, S. Li et Y. Zeng, «Forest fire spread simulating model using cellular automaton with extreme learning machine,» Ecological Modelling, vol. 348, pp. 33-43, 2017.

[15] S. Lauf, D. Haase et B. Kleinschmit, «Land-use scenario modelling based on human decisions--Combining system dynamics and cellular automata,» 2012.

[16] M. Wooldridge, An introduction to multiagent systems, John Wiley \& Sons, 2009.

[17] V. Kocabas et S. Dragicevic, «Bayesian networks and agent-based modeling approach for urban land-use and population density change: a BNAS model,» Journal of geographical systems, vol. 15, pp. 403-426, 2013.

[18] J. Barth $\{1$ 'e $\}$ lemy et T. Carletti, «An adaptive agent-based approach to traffic simulation,» Transportation research procedia, vol. 25, pp. 12381248, 2017.

[19] M. Durak, N. Durak, E. D. Goodman et R. Till, «Optimizing an agentbased traffic evacuation model using genetic algorithms,» chez Winter Simulation Conference (WSC), 2015, 2015.

[20] Dagmar Haase et R. Seppelt, «Modeling and simulating residential mobility in a shrinking city using an agent-based approach,» Environmental Modelling I\& Software, vol. 25, pp. 1225-1240, 2010.

[21] H. Wang et C.-J. Chang, «Simulation of Housing Market Dynamics: Amenity Distribution and Housing Vacancy,» chez Proceedings of the 2013 Winter Simulation Conference: Simulation: Making Decisions in a Complex World, Piscataway, 2013.

[22] P. M. Torrens, «Cellular automata and multi-agent systems as planning support tools,» chez Planning support systems in practice, Springer, 2003, pp. 205-222.

[23] E. Elalaouy, K. Rhoulami et M. D. Rahmani, «Towards an agent based model for simulating residential mobility and urban expansion,» Proceedings of the Mediterranean Conference on Information I\& Communication Technologies. Springer International Publishing, 2016. p. 343-351., 2015.

[24] E. Elalaouy, K. Rhoulami et M. D. Rahmani, «A Novel Modeling based Agent Cellular Automata for Advanced Residential Mobility Applications,» INTERNATIONAL JOURNAL OF ADVANCED COMPUTER SCIENCE AND APPLICATIONS, vol. 8, pp. 337-343, 2017. 